\title{
Bildgebende Diagnostik gynäkologischer Notfälle
}

\section{Imaging of gynecologic emergencies}

\author{
M. W. Wagner, T. A. G. M. Huisman, R. A. Kubik
}

\section{Übersicht}

Einleitung

Methoden

US-/CT-/MRT-Kontrastmittel

in der Stillzeit

Gynäkologische Krankheitsbilder

\section{Zusammenfassung}

Das akute Abdomen ist ein häufiges Krankheitsbild in der Notfalldiagnostik. Pathologische Veränderungen der weiblichen Genitalorgane spielen dabei eine wichtige Rolle. Gynäkologische

\section{Einleitung}

Akute Erkrankungen der weiblichen Genitalorgane sind eine häufige Ursache des akuten Abdomens [1]. Aufgrund der vielen möglichen Differenzialdiagnosen reicht die klinische Untersuchung oft genug nicht aus, um gynäkologische und gastrointestinale Notfälle (z.B. die Appendizitis) zu unterscheiden. Eine zusätzliche diagnostische Herausforderung ist die Abklärung von Notfällen während der Schwangerschaft. Häufige Befunde einer normal verlaufenden Schwangerschaft wie Übelkeit, Erbrechen oder unspezifische Schmerzen beeinflussen die klinische Präsentation und verkomplizieren das klinische Bild. Darüber hinaus ist die klinische Untersuchung einer schwangeren Patientin schwieriger beurteilbar und Laborparameter wie CRP und Leukozyten sind im Rahmen der Schwangerschaft erhöht. Dadurch gewinnt die Bildgebung bei der notfalldiagnostischen Abklärung immer mehr an Bedeutung, was sich nicht zuletzt an den stetig steigenden Untersuchungszahlen zeigt: In den USA hat sich die Häufigkeit des Einsatzes bildgebender Diagnostik

Notfälle gefährden die Fertilität und sind potenziell lebensbedrohlich. Bei der Diagnostik sind viele Differenzialdiagnosen je nach Alter der Patientin und einer eventuellen Schwangerschaft zu beachten. Insbesondere akute gastrointestinale Beschwerden lassen sich in der klinischen Untersuchung oft nicht von gynäkologischen Notfällen unterscheiden. Die Bildgebung leistet hier einen wesentlichen Beitrag zur Einengung der Differenzialdiagnose, zur Therapieentscheidung und zum Therapiemonitoring. Im vorliegenden Übersichtsartikel soll der zentrale Stellenwert der Bildgebung im Rahmen häufiger gynäkologischer Notfälle erörtert werden.

Generell ist die Bildgebung schnell verfügbar, kann die klinische Verdachtsdiagnose objektivieren, und Notfälle, wenn nötig, auch im Verlauf beobachten. Eine detaillierte Anamnese (z.B. Voroperationen, frühere Schwangerschaften) und eine ausführliche klinische Untersuchung stehen jedoch am Anfang jeder Abklärung. Zielführend eingesetzt, kann die radiologische

Diagnostik Verdachtsdiagnosen bestätigen und aus-

während der Schwangerschaft innerhalb der letzten

In Abhängigkeit von der Verdachtsdiagnose kommen verschiedene Verfahren der Bildgebung zum Einsatz:

- Der Ultraschall (US) ist initial die bildgebende Methode der Wahl, unabhängig davon, ob eine Schwangerschaft vorliegt oder nicht.

- Die CT hat ihre Bedeutung bei der Abklärung akuter Traumata.

- Die MRT kommt bei unklaren und subakuten Fällen oder für die Diagnostik in der Schwangerschaft zum Einsatz [3]. 
schließen oder auf andere zugrunde liegende Veränderungen hinweisen.

Dieser Übersichtsartikel soll

- einen Überblick über die unterschiedlichen Erscheinungsbilder gynäkologischer Notfälle im Ultraschall (US), in der CT und in der MRT geben,

- die bildgebende Methode der Wahl je nach klinischer Verdachtsdiagnose empfehlen,

- bei der Interpretation der Erkenntnisse aus der Bildgebung im klinischen Kontext zur Gewährleistung einer adäquaten Patientenversorgung helfen und

- auf die aktuellen Richtlinien zum Gebrauch von Kontrastmittel während der Schwangerschaft und Stillzeit hinweisen.

\section{Methoden}

\section{Ultraschall}

Indikationen. Den Ultraschall (US) zeichnet eine hohe Sensitivität bei vielen akuten Erkrankungen aus. Daneben ist er kosteneffizient und schnell verfügbar, sodass er häufig zur Erstuntersuchung der Patientin eingesetzt wird. Den transabdominellen Ultraschall führt dabei i.d. R. der Radiologe durch. Je nach Habitus der Patientin ist damit eine Aussage über den Uterus und die Adnexe möglich, vor allem aber über pathologische Veränderungen wie freie Flüssigkeit im Abdomen. Differenzialdiagnosen wie eine Harnstauung oder eine Appendizitis können ausgeschlossen werden. Eine genauere Beurteilung der Genitalorgane selbst wird mit der transvaginalen Sonografie erreicht [4]. Mithilfe des Farbdopplers können signifikante Zusatzinformationen gewonnen werden. Bei der Frage nach der Organperfusion ist der Farbdoppler z.B. von entscheidender Bedeutung.

Schwangerschaft. Es sind keine negativen Effekte der Sonografie für die Mutter oder den Fötus bekannt. Die US-amerikanische Food and Drug Administration (FDA) empfiehlt während der Schwangerschaft jedoch eine maximale Schallwellenintensität von $720 \mathrm{~mW} / \mathrm{cm}^{2}$. Diese Intensität kann bei einer Doppler-Untersuchung durchaus erreicht werden. Deshalb sollte der DopplerUltraschall so kurz wie möglich eingesetzt werden gerade so lange, bis die Diagnose gestellt werden kann [5].
Kontrastmittel. Kontrastmittel für die Sonografie bestehen aus gasgefüllten Mikrobläschen, die von einer elastischen Hülle umgeben sind. Diese Hülle besteht aus Lipiden, Albumin, Polymeren oder auch weiteren Hilfsstoffen wie Tensiden oder Polyethylenglykol. Meist werden die Kontrastmittel intravenös appliziert und sind lungengängig. Primär verteilen sie sich in den Gefäßen und weisen damit hohe Kontrastabgrenzung gegenüber dem Extravaskulärraum auf. Sie sind äußerst gut verträglich, werden aber derzeit weder bei Kindern noch bei Schwangeren eingesetzt $[6,7]$. Bei gynäkologischen Notfällen werden i.d.R. keine Ultraschallkontrastmittel angewendet.

\section{Computertomografie}

Indikationen. Aufgrund der Strahlenbelastung sollte die CT vermieden werden - vor allem bei jüngeren und bei schwangeren Patientinnen. Die Wahrscheinlichkeit teratogener Effekte durch ionisierende Strahlen steigt mit der applizierten Dosis. Moderne CT-Protokolle („low dose-CT“) erreichen jedoch nicht die für teratogene Effekte ermittelten Grenzwerte. Vor jeder CTUntersuchung ist eine strenge Nutzen-Risiko-Analyse für die Mutter und den Fötus erforderlich. Keinesfalls sollte die CT als Suchmethode bei unklaren Befunden eingesetzt werden. In einer akut lebensbedrohlichen Situation ist sie dagegen die Methode der ersten Wahl.

Kontrastmittel in der Schwangerschaft. In Tierstudien hatten wasserlösliche, jodhaltige Kontrastmittel bei intravenöser Applikation keinen teratogenen Effekt. Die parenterale Injektion, insbesondere nach der 12. Schwangerschaftswoche, erhöht jedoch das Risiko einer neonatalen Hypothyreose. Daher empfehlen die Richtlinien der European Society of Urogenital Radio$\operatorname{logy}$ (ESUR) [5,8]:

- Jodhaltiges Kontrastmittel nur applizieren, wenn es unbedingt nötig ist.

- In der ersten Woche nach der Geburt ein Screening für neonatale Hypothyreose durchführen.

Unabhängig vom Schwangerschaftsstatus oder von Alter und Geschlecht des Patienten sollte man sich vor jeder Bildgebung fragen, ob ionisierende Strahlung zur Beantwortung der Fragestellung benötigt wird, Kontrastmittel entscheidend ist und die Ergebnisse der Bildgebung die Therapie ändern. 


\section{Magnetresonanztomografie}

Indikationen. Bei der Diagnostik des weiblichen Beckens ist die MRT der CT als Ergänzung zum Ultraschall in mehrfacher Hinsicht überlegen: Die Strahlenbelastung fehlt, der Weichteilkontrast ist höher und man kann verschiedene Kontraste erhalten. Bis heute gibt es keine Hinweise auf ein erhöhtes Risiko für den Fötus bei einer MRT mit 1,5T oder geringerer Magnetfeldstärke. Aufgrund eingeschränkter Datenlage gilt dies jedoch nur für das 2. und 3. Trimenon. Vorsichtshalber sollte deshalb eine MRT im 1. Trimenon vermieden bzw. - je nach maternaler Indikation - besonders sorgfältig abgewogen werden. Die Sicherheit von 3TGeräten wurde zwar noch nicht hinreichend bestätigt, jedoch liegen keine Berichte über nachteilige Effekte vor. Tierversuche mit stärkeren Magneten (>3T) und verlängerter Expositionszeit ergaben keine teratogenen Effekte [5]. Dessen ungeachtet sollte man Schwangere vor jeder MRT- oder CT-Untersuchung ausführlich aufklären und diese Aufklärung schriftlich dokumentieren.

Kontrastmittel in der Schwangerschaft. Die Datenlage ist zwar eingeschränkt, aber es scheint so, als ob die Applikation gadoliniumhaltiger Kontrastmittel keine Nachteile für den Fötus hätte. Gadolinium überschreitet die Plazentabarriere und wird über die fötalen Nieren in die Amnionflüssigkeit ausgeschieden. Die FDA empfiehlt den Einsatz von gadoliniumhaltigem Kontrastmittel nur bei positivem Nutzen-Risiko-Verhältnis. Es sollte dann die geringstmögliche Dosis eines stabilen, makrozyklischen Kontrastmittels verwendet werden (Tab. 1) [5, 8]. Eine korrekte Diagnosestellung ist jedoch in den meisten Fällen auch ohne Kontrastmittel möglich, sodass wir während der Schwangerschaft kein Kontrastmittel verabreichen.

Bei gynäkologischen Notfällen sind US und MRT grundsätzlich die Methoden der Wahl. Wenn die $C T$, z. B. bei einem akuten Abdomen, zum Einsatz kommt, sollte die Strahlendosis möglichst niedrig bleiben (ALARA-Prinzip). Die MRT ist subakuten Krankheitsbildern vorbehalten und wird vorwiegend zur Abklärung sonografisch unklarer Befunde eingesetzt. Ein positives Nutzen-Risiko-Verhältnis kann in seltenen Fällen den Einsatz von CT und MRT sowie den Gebrauch von Kontrastmitteln in der Schwangerschaft rechtfertigen.

\section{Tabelle 1}

Strahlendosis und Kontrastmittel in Schwangerschaft und Stillzeit

\begin{tabular}{|c|c|}
\hline Phase & Strahlendosis und Kontrastmittel \\
\hline Schwangerschaft $[8,77]$ & $\begin{array}{l}\text { CT } \\
\text { - ionisierende Strahlung } \\
\text { - teratogenes Risiko minimal bei fötaler Dosis } \\
<100 \text { mGy } \\
\text { - gesteigertes relatives Risiko für Neoplasie mit } \\
\text { Todesfolge } \\
\text { - jodhaltige Kontrastmittel } \\
\text { - in Ausnahmefällen - nur wenn absolut indiziert } \\
\text { - Überprüfung der Schilddrüsenfunktion des Neu } \\
\text { geborenen innerhalb der ersten Woche nach Geburt } \\
\text { MRT } \\
\text { - relative Kontraindikation im 1. Trimester } \\
\text { gadoliniumhaltige Kontrastmittel } \\
\text { - in Ausnahmefällen - nur wenn absolut indiziert } \\
\text { - kleinstmögliche Dosis des stabilsten makrozyklischen } \\
\text { Kontrastmittels } \\
\text { - keine weiteren Tests bei Neugeborenen notwendig } \\
\text { - bei eingeschränkter Nierenfunktion: keine gado- } \\
\text { liniumhaltigen Kontrastmittel anwenden }\end{array}$ \\
\hline Stillzeit $[8,77]$ & $\begin{array}{l}\text { - CT: nach jodhaltigem Kontrastmittel kann unverändert } \\
\text { weiter gestillt werden. } \\
\text { - MRT: bei stabilen, makrozyklischen Gadoliniumpräpa- } \\
\text { raten kann unverändert weiter gestillt werden. }\end{array}$ \\
\hline
\end{tabular}

\section{US-/CT-/MRT-Kontrastmittel in der Stillzeit}

\section{Ultraschall-Kontrastmittel}

Das Stillen kann nach Gabe der im klinischen Alltag nur selten zwingend indizierten Ultraschall-Kontrastmittel normal fortgesetzt werden.

\section{Jodierte und gadoliniumhaltige Kontrastmittel}

Jodierte und gadoliniumhaltige Kontrastmittel werden zu ca. 0,5\% der verabreichten Dosis innerhalb der ersten 24 Stunden über die Muttermilch ausgeschieden. In der Folge wird weniger als $1 \%$ der vom Kind mit der Muttermilch aufgenommenen Kontrastmittelmenge im Darm des Kindes resorbiert. Die Belastung des Neugeborenen ist somit zu vernachlässigen. Bezüglich der Jodidbelastung liegen keine Berichte über eine erhöhte Sensibilisierung, Allergieraten oder von Spätreaktionen bei Neugeborenen vor. 
Bei Einsatz von Gadolinium in der Stillzeit werden die stabileren, makrozyklischen Komplexe empfohlen. Nach den Richtlinien der ESUR kann danach unverändert weiter gestillt werden [5].

Bei den linearen, instabileren Gadoliniumpräparaten empfehlen die ESUR-Leitlinien, für mindestens 24 Stunden nach Kontrastmittelgabe nicht zu stillen. Die Studie von Kubik et al. zeigte jedoch auf, dass die Muttermilch bei parenteraler Applikation eines linearen Gadoliniumpräparats über länger als 48 Stunden gadoliniumhaltig ist [9]. Demnach genügt eine Stillpause von lediglich 24 Stunden nicht, um eine systemische Belastung des Neugeborenen durch das lineare Kontrastmittel zu vermeiden [5,8]. Daraus ergibt sich, dass makrozyklisches Gadolinium während der Schwangerschaft bevorzugt werden sollte.

\section{Gynäkologische Krankheitsbilder}

\section{Hämatometrokolpos}

Beim Hämatometrokolpos (HMK) sind Vagina und Uterus distendiert und blutgefüllt. Die Inzidenz des HMK liegt bei Teenagern bei etwa 1:1000-2000. Etwa zwei Drittel aller Fälle sind Folge einer Hymenalatresie. Andere mögliche Ursachen sind kloakale Fehlbildungen und Anomalien der Müller-Gänge, z.B. ein transversales Septum bei einem Uterus didelphys [10]. Die Diagnose eines HMK wird zumeist im Rahmen der Pränataldiagnostik, direkt nach der Geburt oder mit Beginn der Pubertät gestellt. Typische Symptome zu Beginn der Pubertät sind Bauchmerzen in Verbindung mit primärer Amenorrhö [11,12].

Ultraschall. Im Ultraschall lässt sich der Uterus mit gemischt echogener, mehrheitlich echoreicher intraluminaler Flüssigkeit abgrenzen. Bei ausgeprägten Befunden kann auch eine Hydro-/Hämatosalpinx auftreten [12-14]. Bei der US-Untersuchung sollte man auch nach einer assoziierten Nierenfehlbildung oder einer Hydronephrose suchen. In der Regel verwendet man den transabdominellen Ultraschall, da der transvaginale Ultraschall bei Virgos nicht durchgeführt wird. Eine mögliche, aber nur selten eingesetzte Alternative ist der transrektale Ultraschall.
Die Sonografie zeigt beim HMK einen dilatierten Uterus, der mehrheitlich echoreiche Flüssigkeit enthält. Zeitgleich sollten eine assoziierte Nierenfehlbildung und eine Hydronephrose ausgeschlossen werden.

CT. Die CT wird oft initial auf der Suche nach den Ursachen der akuten Bauchschmerzen eingesetzt. US und MRT sind bei der Verdachtsdiagnose eines HMK jedoch zu bevorzugen. In der CT ist ein HMK eine mehr oder weniger hyperdense, nicht kontrastmittelaufnehmende, flüssigkeitsgefüllte Raumforderung. Die Ausdehnung kann bis zu $25 \mathrm{~cm}$ betragen [15].

MRT. Die MRT ist aufgrund des besseren Weichteilkontrasts die Methode mit der höchsten Aussagekraft. Sie ist vor allem indiziert, wenn der Verdacht auf eine komplexe Fehlbildung besteht oder ein chirurgischer Eingriff geplant wird $[12,13,16]$. Je nach Alter der Hämorrhagie variiert die Signalintensität des Blutes. Im subakuten Stadium ist Blut auf T1w und T2w Aufnahmen in der Regel hyperintens, im chronischen Stadium dagegen hypointens (Abb. 1, Abb. 2, Abb. 3).

Die MRT ist bei einem HMK indiziert, wenn der Verdacht auf komplexe Fehlbildungen besteht oder ein chirurgischer Eingriff vorbereitet werden soll.

\section{Adnextorsion}

Die Torsion der Adnexe ist ein seltener Notfall. Er betrifft 2,7-7,4\% aller Frauen mit akutem Unterleibsschmerz $[17,18]$. Etwa $71 \%$ aller Adnextorsionen treten zwischen dem 20. und 39. Lebensjahr auf [19]. Die Torsion verhindert zunächst den venösen und lymphatischen Rückfluss und beeinträchtigt später auch die arterielle Versorgung der Adnexe. Mögliche Folgen sind Thrombosierung, Ischämie und hämorrhagische Infarzierung der Adnexe [20,21]. Für den Erhalt der Fertilität ist eine frühe Diagnose von herausragender Bedeutung. Adnextorsionen sind auf der rechten Seite häufiger (55-71\%) und betreffen sowohl das Ovar als auch die Tuba uterina (72\%) [22,23]. In 50-90\% aller Fälle liegt der Torsion eine ovarielle Raumforderung zugrunde:

- Teratome $(17,2 \%)$

- paratubale Zysten oder Hydrosalpinx (16,7\%)

- Follikelzysten (15,9\%)

- Zystadenome (13,8\%)

- außerdem (seltener): Corpus-luteum-Zysten, primäre oder sekundäre Tumoren, Adhäsionen oder Extrauteringravidität (EUG) 
Bei Kindern handelt es sich zumeist um eine hypermobile Adnexe ohne zugrunde liegende Erkrankung. Die Torsionsgefahr steigt signifikant bei Ovarialzysten ab einer Poldistanz von $5 \mathrm{~cm} \mathrm{[18].}$

Ultraschall. Mit der sonografischen Primärdiagnostik kann in 46-74\% die Diagnose gestellt werden [24]. Zeichen einer Adnextorsion sind vergrößerte Ovarien $(>4 \mathrm{~cm})$ mit randständigen Follikeln und freier Flüssigkeit im Douglas-Raum [21,23,25]. Die randständige perlschnurartige Anordnung der Follikel wird „stringof-pearls"-Zeichen genannt und kommt in 12-74\% aller Fälle vor. Duplexsonografisch lässt sich häufig (13-88\%) ein „Whirlpool“-Zeichen nachweisen, das durch die torquierten arteriellen und venösen Gefäße versursacht wird. Das ovarielle Doppler-Signal ist jedoch fehleranfällig: Es war in 45-61\% aller Fälle mit operativ bestätigter Adnextorsion normal (Abb.4) [20,21, 25-27].

CT. Bei einer Torsion ist die CT dem Ultraschall diagnostisch unterlegen [23]. Für eine Torsion sprechen vergrößerte, wandverdickte Adnexe, Aszites, eine Deviation des Uterus nach ipsilateral sowie eine angrenzende Fettgewebsimbibierung [23, 28, 29].

MRT. Die MRT ist bei Patientinnen mit subakuten Symptomen und unklaren US-Befunden indiziert [21, 30]. Ihr Weichteilkontrast ist im Vergleich zur CT höher, sodass sich freie Flüssigkeit im Douglas-Raum, vergrößerte Ovarien $(>4-5 \mathrm{~cm}$ ) mit randständigen Follikeln, periovarielle Ödeme und Hämatome sowie die hämorrhagische Infarzierung torquierter Adnexe besser darstellen [24,27]. Die Befunde mit der höchsten Sensitivität in der MRT sind die Wandverdickung der Tuba uterina (91\%) und ein „Whirlpool“-Zeichen (68\% aller Fälle) [17]. Was den zusätzlichen Nutzen der diffusionsgewichteten Bildgebung bei Adnextorsionen betrifft, fehlen aktuell noch größere Studien. In einer Studie mit 12 Patientinnen ging die hämorrhagische Infarzierung der torquierten Adnexraumforderungen mit einer signifikant erhöhten Diffusionsrestriktion der Wand der Raumforderungen einher [31].

\section{Pelvic inflammatory disease}

Die „pelvic inflammatory disease“ (PID) ist ein akutes klinisches Syndrom, das durch eine aufsteigende Infektion von Mikroorganismen (z.B. Chlamydien, Neisserien) verursacht wird. Die PID beginnt in der Vagina oder der Zervix und aszendiert über das Endometrium in die Tuba uterina und zum Ovar. Etwa ein Drittel aller
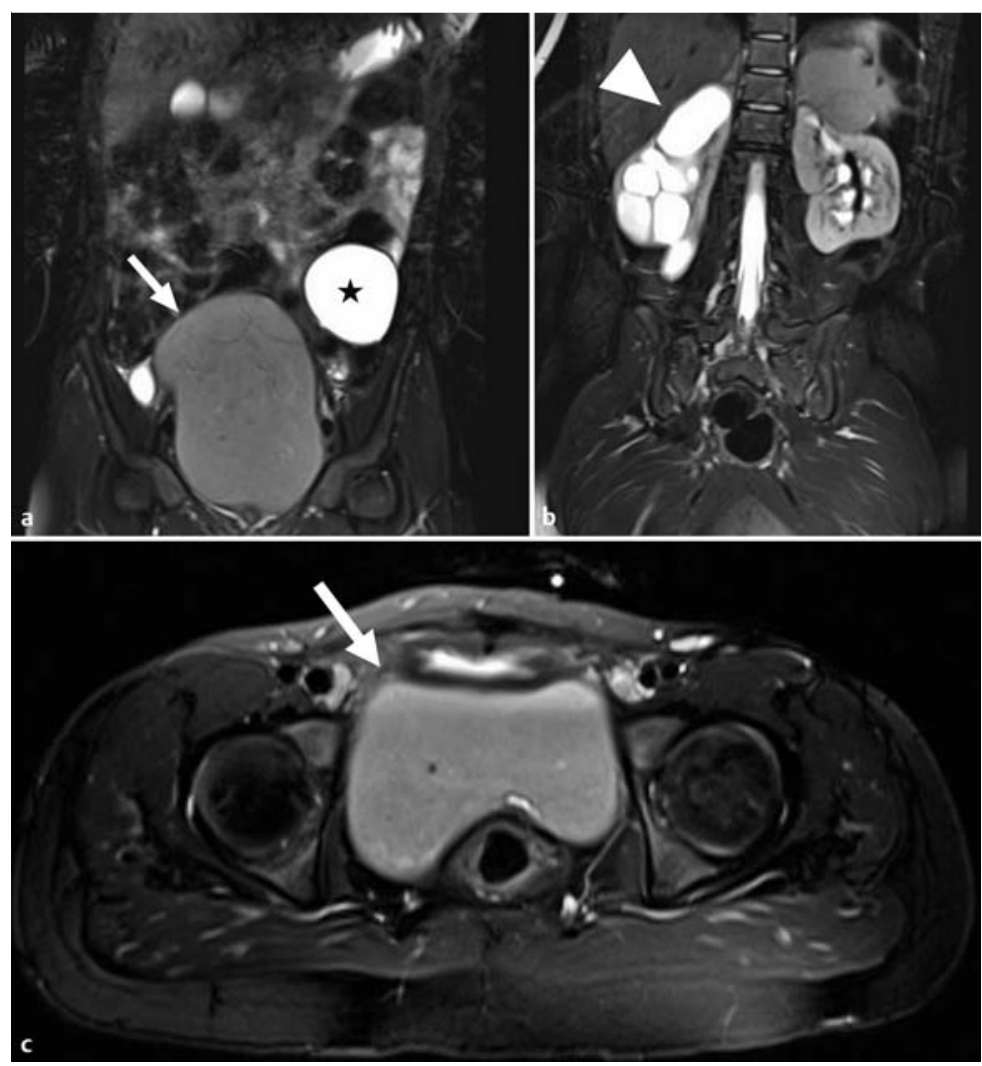

Abb. 112 Jahre alte Patientin mit kloakaler Fehlbildung. Der Pfeil zeigt eine isointense Flüssigkeitsakkumulation in der T2w Sequenz in dilatierter Vagina und Uterus. Die Pfeilspitze weist auf eine assoziierte Hydronephrose. Der Stern markiert eine inzidentelle Nierenzyste links. a, b Fettgesättigte T2w Sequenz in koronarer Schichtführung. c Fettgesättigte T2w Sequenz in axialer Schichtführung.

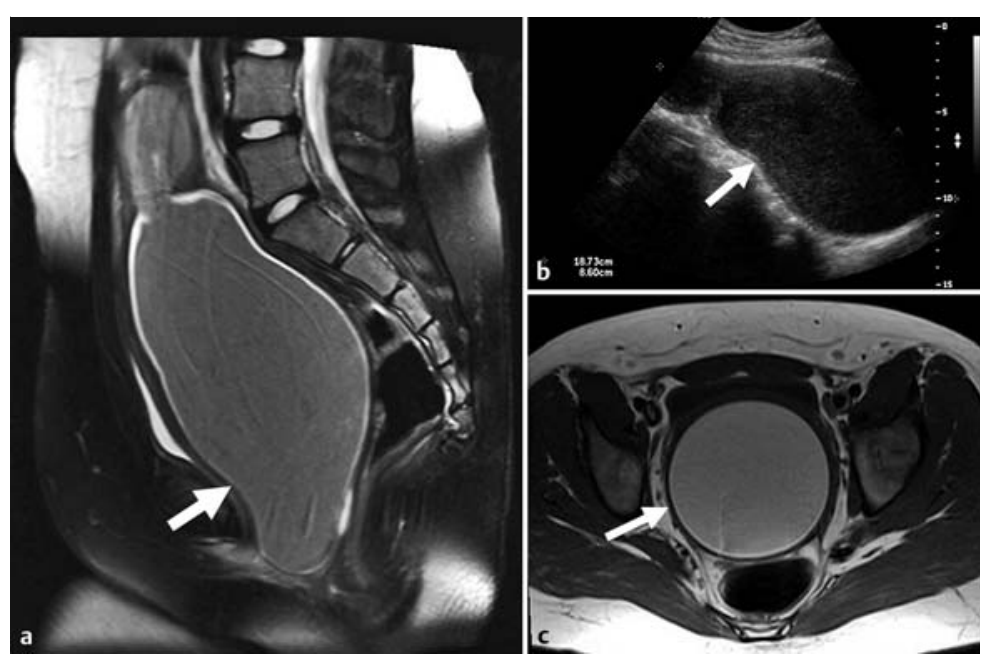

Abb. 211 Jahre alte Patientin mit Hämatokolpos bei Hymenalatresie. Der Pfeil weist auf eine hypoechogene bzw. in der T1w Sequenz hyperintense, in der T2w Sequenz isointense Flüssigkeitsakkumulation in der dilatierten Vagina. a Sagittale T2w Sequenz. b Transabdomineller Ultraschall. c Axiale, T1w Sequenz. 

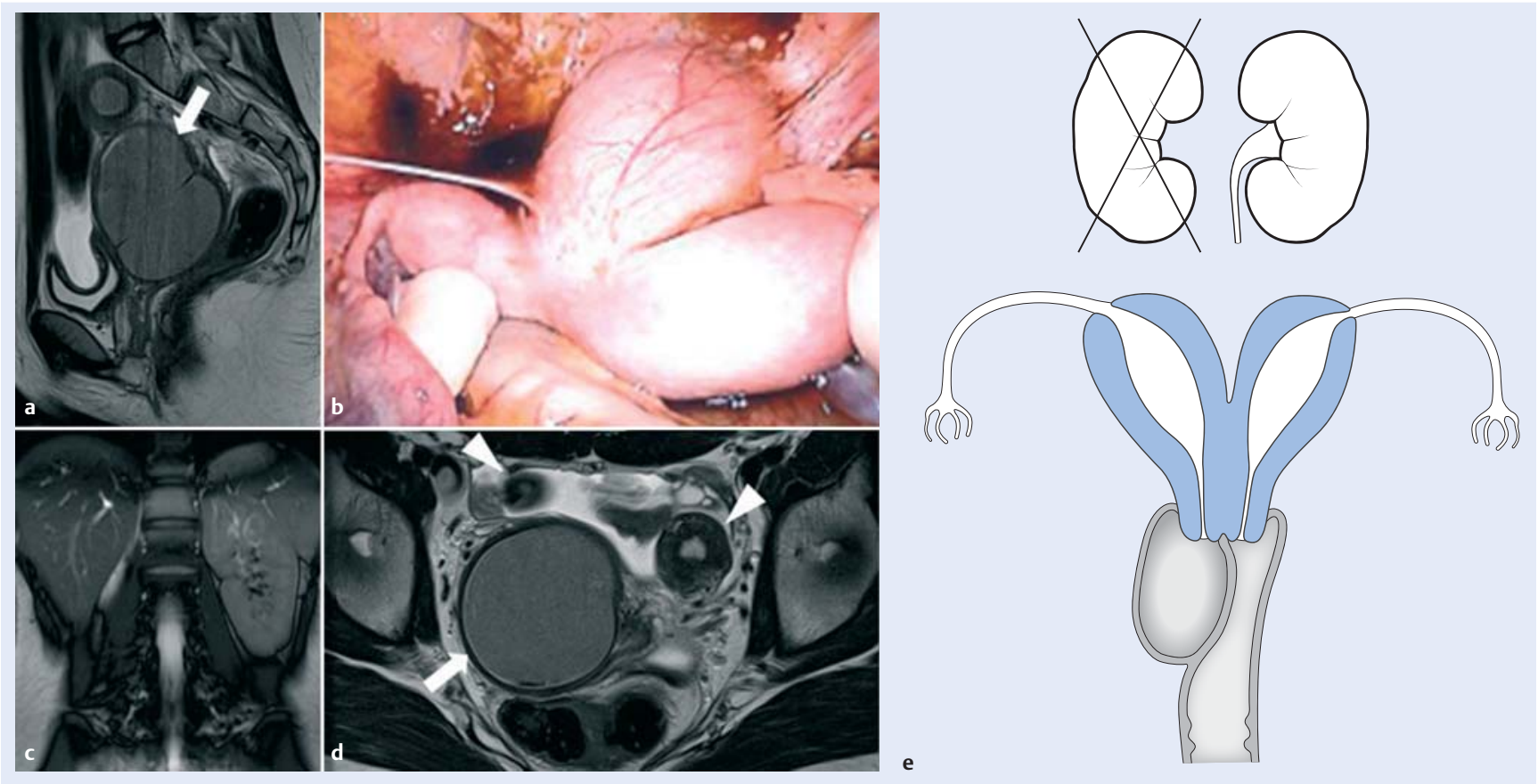

Abb. 313 Jahre alte Patientin mit Uterus didelphys, Nierenagenesie rechts und transversalem vaginalem Septum mit Hämatometrokolpos. Der Pfeil markiert den Hämatometrokolpos, die Pfeilspitzen in d den Uterus didelphys. a Sagittale, T2w Sequenz. b Intraoperativer Situs mit dilatierter Vagina. c Koronare TrueFISPSequenz. Nierenagenesie rechts. d Axiale TrueFISP-Sequenz. e Schema der Fehlbildungen: Uterus didelphys, Nierenagenesie rechts und transversales vaginales Septum (aus [75]).

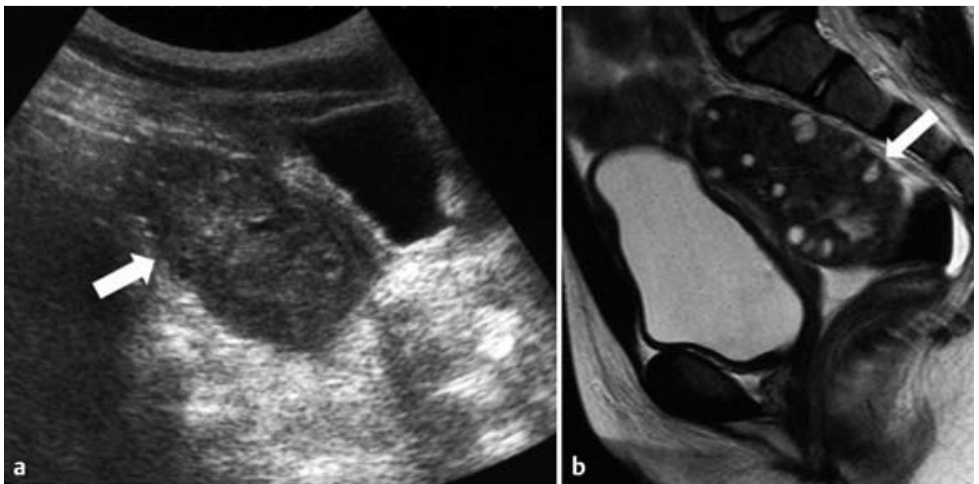

Abb. 46 Jahre alte Patientin mit Adnextorsion. a Transabdomineller Ultraschall. Der Pfeil weist auf die überwiegend hypoechogene Raumforderung kraniodorsal der Harnblase mit dorsaler Schallverstärkung. b Sagittale T2w Sequenz. Der Pfeil zeigt das vergrößerte Ovar mit randständigen Follikeln. Zusätzlich findet sich etwas Flüssigkeit in der Excavatio rectouterina. bewegungsabhängige Schmerzen der Cervix uteri können dabei aufschlussreich sein.

Ultraschall. Der US ist für den Nachweis eines TOA in retrospektiven Studien hochsensitiv (83-100\%) und spezifisch $(83-98,6 \%)$ [34]. Übliche Befunde sind solide und/oder zystische Raumforderungen sowie freie Flüssigkeit in der Adnexregion und im DouglasRaum. Darüber hinaus verliert der Uterus seine scharfe äußere Kontur und das endometriale Echo kann fehlen [35].

Im US erkennt man bei einem TOA solide und/oder zystische Raumforderungen sowie freie Flüssigkeit in der Adnexregion und im Douglas-Raum.

CT. In der CT erscheinen TOA als wandverdickte Flüssigkeitskollektionen mit randständiger Kontrastmittelaufnahme [32,35-37]. Die Mesosalpinx ist verdickt und weicht nach ventral ab $(91-100 \%)[32,35]$. Die Fettlamelle zwischen Abszess und angrenzenden Organen (Rektum, Colon sigmoideum, Ureter) ist verstrichen (58-91\%) [32,33,36,37]. Für einen TOA sprechen außerdem verdickte Ligg. uterosacralia ( $45-66 \%$ ), eine Pyosalpinx (50\%) und extraluminale freie Luft $(12,5 \%)$ [32,35-37]. 

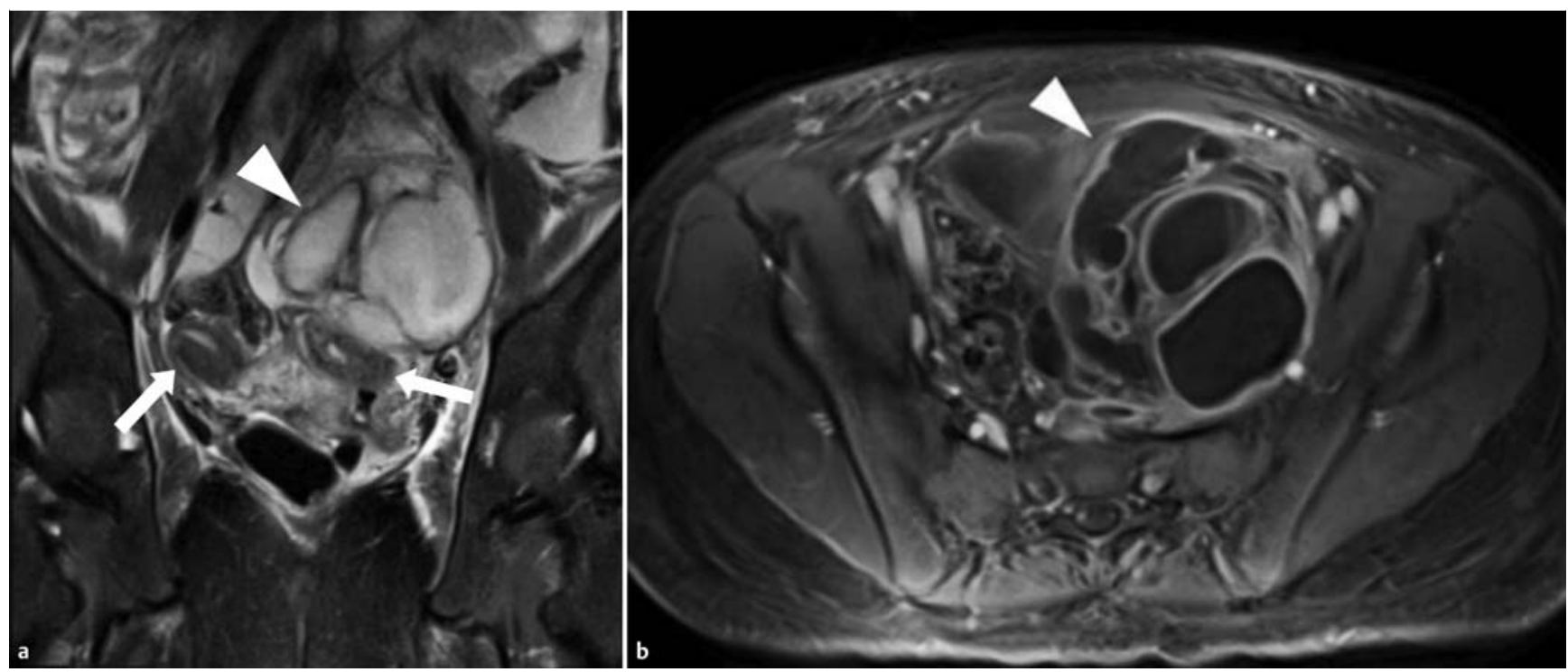

Abb. 515 Jahre alte Patientin mit Uterus didelphys und Tuboovarialabszess. Die Pfeilspitze weist auf die randständig kontrastmittelaufnehmende, polylobulierte Raumforderung, die dem Tuboovarialabszess entspricht. a Koronare, fettgesättigte T2w Sequenz. Die Pfeile markieren den Uterus didelphys. b Axiale, kontrastmittelverstärkte, fettgesättigte T1w Sequenz.

Hilfreich für die Differenzialdiagnose von TOA und Ovarialkarzinom ist das klinische Bild der Patientin (z.B. Entzündungszeichen) und die MRT.

MRT. Die MRT hat die höchste Sensitivität und Spezifität für abdominelle Abszesse (100\% bzw. 94\%) [38]. Der TOA ist i.d.R. hypointens auf T1w und gemischt hyperintens auf T2w Aufnahmen [39,40]. Die Abszesskapsel lässt sich am besten auf der kontrastmittelverstärkten, fettgesättigten T1w Sequenz erkennen [38]. Die Signalintensität des Abszessinhalts hängt von ihrer Proteinkonzentration ab: Häufig findet sich etwas hyperintenses hämorrhagisches Granulationsgewebe im Inneren auf der T1w Sequenz [40]. Die i.d. R. unscharf demarkierte Ausbreitung der entzündlichen Reaktion ist auf T2w Aufnahmen hyperintens und auf der fettgesättigten, kontrastmittelverstärkten T1w Aufnahmen isointens [39]. Zur Differenzialdiagnose des Ovarialkarzinoms mithilfe der diffusionsgewichteten Bildgebung ist die Datenlage bislang nicht einheitlich (Abb.5) $[41,42]$.

Die MRT ist die beste Methode zur Diagnostik intraperitonealer Abszesse. Der Abszess ist hypointens auf T1w und hyperintens auf T2w Aufnahmen. Für die perifokale entzündliche Reaktion sind die T2w Sequenz und die kontrastmittelverstärkte, fettgesättigte T1w Sequenz hilfreich.

\section{Postpartale Ovarialvenenthrombose}

Die postpartale, septische Ovarialvenenthrombose (SPOVT) ist eine weitere, seltene Komplikation der PID. Die Prävalenz liegt bei 0,15-0,18\% innerhalb der postpartalen Population [43]. Die Inzidenz liegt bei 1:9000 nach Vaginalgeburt und 1:800 nach Sectio caesarea [44]. Es können grundsätzlich beide Vv. ovaricae thrombosieren, die rechte Seite ist jedoch häufiger betroffen [43]. Die SPOVT ist häufig eine Ausschlussdiagnose bei postpartal persistierendem Fieber. Therapeutisch werden Breitspektrumantibiotika parenteral appliziert; systemisch wird mit niedermolekularem Heparin antikoaguliert $[45,46]$.

Ultraschall. Mithilfe des US lässt sich das vergrößerte Ovar mit einer angrenzenden dilatierten tubulären Struktur abgrenzen. Postpartal sind dilatierte Ovarialvenen häufig, deshalb sollte man mit dem Doppler den venösen Fluss kontrollieren. Bei unklaren Befunden oder fehlendem Flusssignal sind eine CT oder eine MRT indiziert [47]. Die Ovarialvenen sind in ihrem Verlauf schwierig kontinuierlich darzustellen, die proximale Insertion an die V. cava inferior kann jedoch häufig eingesehen werden.

\section{CT. In der kontrastmittelverstärkten CT ist die Diagnose} der SPOVT leichter zu stellen. Eine intraluminale, hypodense Struktur in einem aufgeweiteten Gefäß entspricht dem frischen Thrombus. Zusätzlich findet sich eine kontrastmittelaufnehmende Gefäßwand vom Ovar bis zur V. cava inferior auf der rechten bzw. bis zur 

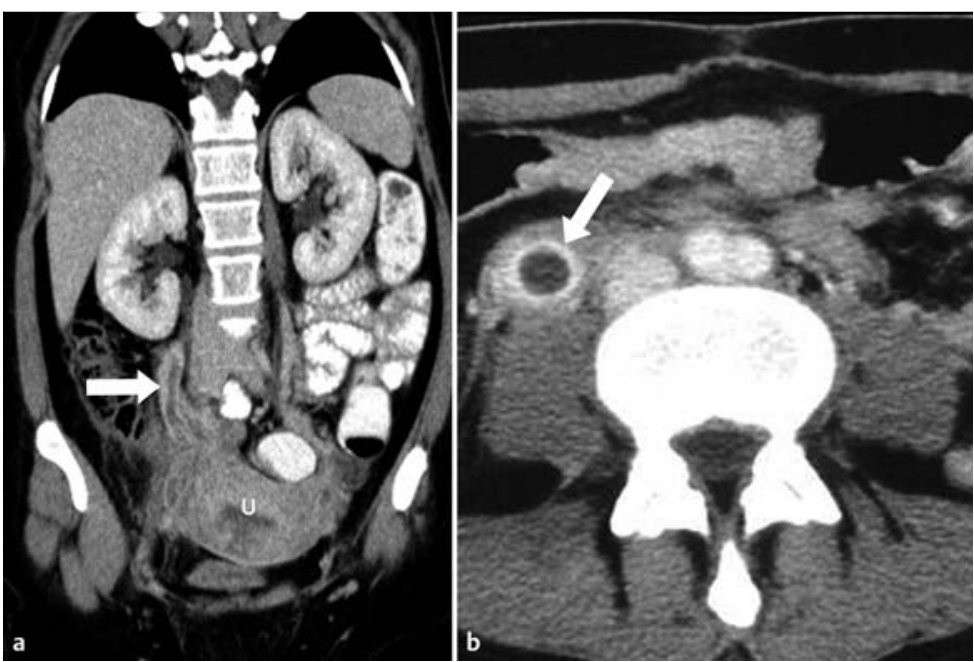

Abb.6 Septische, postpartale Ovarialvenenthrombose (SPOVT). Der Pfeil markiert die dilatierte, randständig kontrastmittelaufnehmende $\mathrm{V}$. ovarica mit intraluminalem hypodensem Füllungsdefekt (=Thrombus) und angrenzendem imbibiertem Fettgewebe. a Koronare Reformatierung eines kontrastmittelverstärkten CTs; U =Uterus. b Axiale Ebene eines kontrastmittelverstärkten CTs.

Nierenvene auf der linken Seite. Eine angrenzende Fettgewebsimbibierung ist möglich (Abb.6).

MRT. In der MRT ist der Thrombus als hypointenser Füllungsdefekt auf kontrastmittelverstärkten, T1w Aufnahmen und hyperintens auf T2w Aufnahmen zu sehen. In der diffusionsgewichteten Sequenz besteht eine Restriktion des Thrombus [48]. Der Vorteil der Diffusionsgewichtung wie auch der Time-of-Flight(TOF-)Angiografie liegt in der Einsparung des Kontrastmittels gegenüber den konventionellen MRTSequenzen und der CT [48].

\section{Extrauteringravidität}

Die Extrauteringravidität (EUG) ist der häufigste Notfall in der frühen Schwangerschaft. Die Diagnose wird üblicherweise durch die Messung des humanen Choriongonadotropins (hCG) und den transvaginalen Ultraschall (TVUS) gestellt. Bei sinkender Sterblichkeitsrate stieg die Inzidenz der EUG in den letzten Jahrzehnten [49]. Risikofaktoren der EUG sind „pelvic inflammatory disease“, Endometriose, vorangegangene gynäkologische Operationen, Infertilitätstherapie, späte Primiparität und assistierte reproduktive Therapien (künstliche Befruchtung, In-vitro-Fertilisation). Nahezu alle EUGs treten in der Tuba uterina auf: $55 \%$ in der Ampulle, 25\% im Isthmus und 17\% in den Fimbrien [50]. Zu den anderen, sehr seltenen Manifestationsorten (ca. 3\% aller Fälle) zählen die Cervix uteri, Narben nach Sectio caesarea, anlagebedingte Rudimente und die Abdominalhöhle [51]. Im Rahmen assistierter reproduktiver Therapien nehmen die selteneren Manifestationsorte der EUG leichtgradig zu (Abb.7) [52,53].

Ultraschall. Aufgrund der sehr hohen Sensitivität und Spezifität (69-99\% bzw. 84-99,9\%), seiner hohen Verfügbarkeit und seiner Kosteneffizienz ist der transvaginale Ultraschall bei der EUG die Methode der Wahl [54-58]. In bis zu 57,9\% aller Fälle lässt sich die EUG als kleine solide, inhomogene Raumforderung („blob“Zeichen) in unmittelbarer Nähe zum Ovar abgrenzen [55]. Ein „tubal-ring“-Zeichen (EUG als hyperechogener Ring) wurde in 20,4\% und ein Gestationssack mit einem fötalen Pol in 13,2\% berichtet [55]. Hämatometra, Hämatoperitoneum oder Patientenunruhe mit starken Schmerzen schränken die Aussagekraft des US jedoch erheblich ein.

Der transvaginale Ultraschall ist bei einer EUG die diagnostische Methode der Wahl. Ultraschallzeichen der EUG sind das „blob-sign“ und das „tubal ring-sign“.

CT. Die CT wird zwar häufig zur Abklärung eines akuten Abdomens angefordert, sie sollte jedoch bei Schwangeren vermieden werden. Die CT-Kriterien einer EUG korrelieren im Wesentlichen mit den US-Befunden.

MRT. Die MRT dient der Abklärung in unklaren Fällen. Die Hämatometra ist ein für die EUG diagnostischer Befund und stellt sich iso-/hyperintens auf T1w Aufnahmen, mit gemischtem Signal auf T2w Aufnahmen und mit Suszeptibilitätsartefakten auf T2*w Aufnahmen dar. Die EUG selbst kommt als solide wirkende Raumforderung isointens in der T1w Sequenz und gemischt iso- bis hypointens in der T2w Sequenz zur Darstellung (Abb. 8) [59].

Die für die EUG typische Hämatometra ist iso-/ hyperintens auf T1w Aufnahmen und zeigt ein gemischtes Signalverhalten auf T2w und Suszeptibilitätsartefakte auf T2*w Aufnahmen.

\section{Leiomyome}

Leiomyome sind benigne Tumoren, die mehrheitlich aus glatter Muskulatur und zu einem variablen Anteil aus fibrösem Bindegewebe bestehen. Leiomyome sind die häufigsten gynäkologischen Neoplasien und treten bei 20 - 30\% aller Frauen im gebärfähigen Alter auf. Je nach Lokalisation unterscheidet man submuköse, intramurale oder subseröse Leiomyome [60]. Submu- 
köse und subseröse Leiomyome neigen zu gestieltem Wachstum mit erhöhtem Risiko für Torsion, Infarkt und Nekrose [61]. Als parasitisch gelten Leiomyome, wenn nach Torsion des vaskulären Pedikels eine Neovaskularisation über extrauterine Strukturen entsteht. Bedingt durch hormonell beschleunigtes Wachstum werden Leiomyome während einer Schwangerschaft häufiger symptomatisch und die Inzidenz der Torsion ist erhöht $[5,62]$. Leiomyome können auf verschiedene Weisen degenerieren. Die mit ca. $60 \%$ aller Fälle häufigste Variante ist die hyaline Degeneration [63]. Aber auch eine zystische, eine myxoide und eine rote Degeneration sind möglich. Letztere präsentiert sich klinisch während der Schwangerschaft mit einem akuten Abdomen und ist gekennzeichnet durch eine hämorrhagische Infarzierung des Leiomyoms.

Leiomyome sind die häufigsten gynäkologischen Neoplasien. In der Schwangerschaft ist die Inzidenz der Torsion des Leiomyoms erhöht. Die rote Degeneration ist eine Differenzialdiagnose für das akute Abdomen während der Schwangerschaft.

Ultraschall. Im US lässt sich eine solide Raumforderung mit niedriger Echogenität abgrenzen. Kalzifikationen erscheinen als hyperechogene Foki mit dorsaler Schallauslöschung [64]. Ein fehlendes Flusssignal in der Doppler-Sonografie weist auf eine Stieldrehung hin [65].

CT. In der CT stellen sich degenerierte und bereits nekrotische Leiomyome zentral hypodens dar. Randständig nehmen sie als Korrelat für torquierte Venen oder dilatierte, lymphatische Gefäße Kontrastmittel auf. Der Infarkt eines Leiomyoms ist als Perfusionsverlust zu erkennen. Eine sich anschließende hämorrhagische Infarzierung erscheint hyperdens in der nativen CT [65]. Typisch ist ein Ödem um das torquierte und infarzierte Leiomyom [66].

MRT. Die MRT hat eine Schlüsselfunktion inne, wenn das Leiomyom im US nur eingeschränkt beurteilt werden kann. Der entscheidende Vorteil der MRT ist die im Vergleich zu anderen Methoden leichtere anatomische Zuordnung des Leiomyoms zum Uterus. In der gestielten Verbindung lässt sich ein „bridging-vascular“-Zeichen nachweisen, das eine Sensitivität von 76,9\% aufweist. Darunter versteht man die Darstellung versorgender, torquierter Gefäße, die eine „vaskuläre Brücke“ zwischen Uterus und Myom bilden. Diese Brücke ist auf T2w und auf kontrastmittelverstärkten T1w Aufnahmen zu erkennen. Unabhängig von einer Stieldrehung kann man ein subseröses Leiomyom von Tumoren

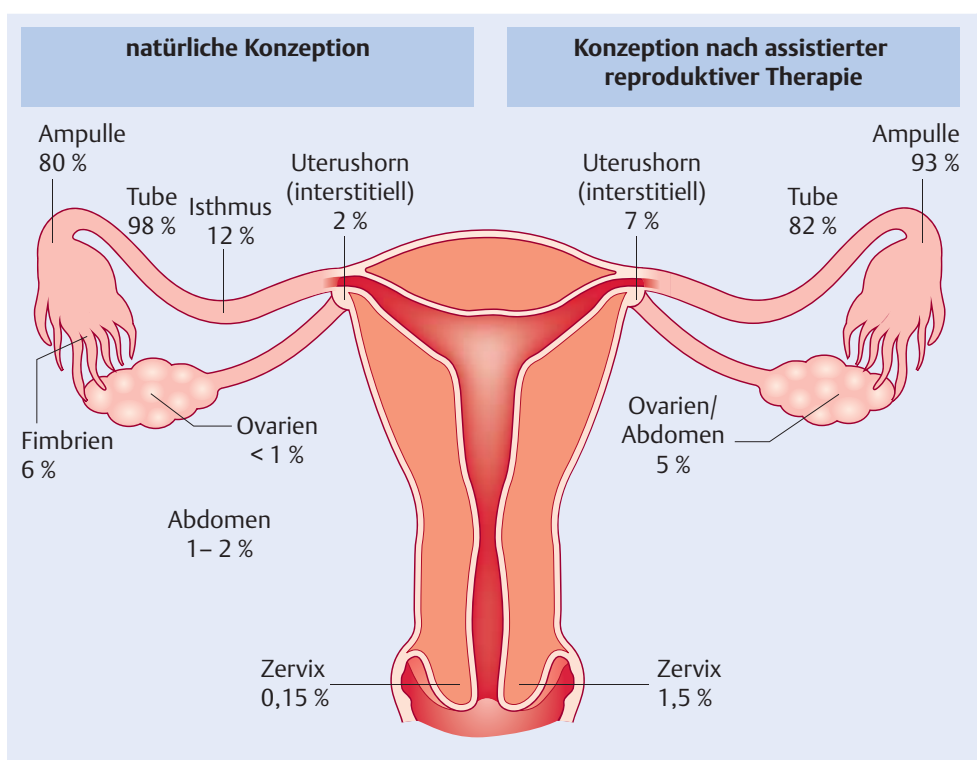

Abb.7 Verteilung der extrauterinen Schwangerschaft mit bzw. ohne assistierte reproduktive Therapie (aus [76]).

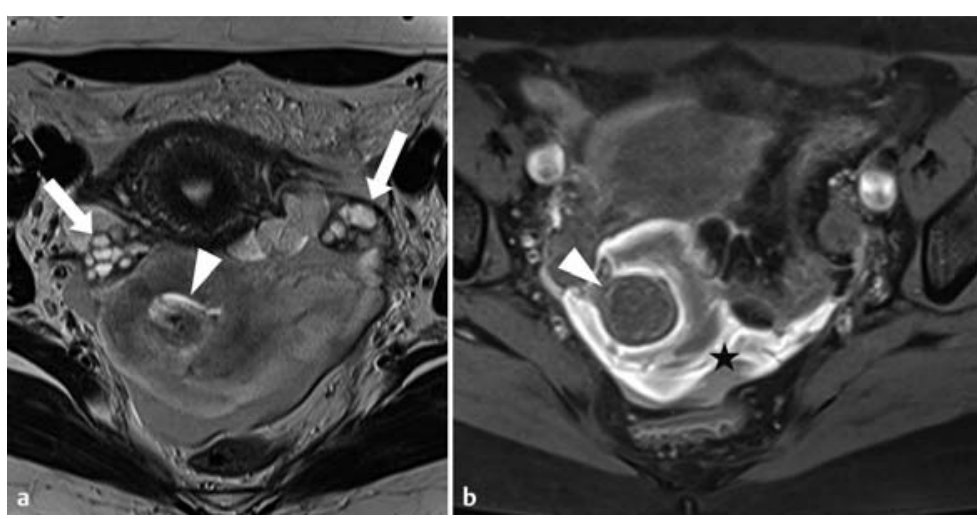

Abb. 8 Rupturierte EUG. Die Pfeilspitze weist auf eine solide, in der T2w Sequenz gemischt iso-/hypointense, in der T1w Sequenz isointense Raumforderung, der EUG entsprechend. Ein perifokales Hämatom ist durch den Stern markiert. a Axiale T2w Sequenz. Die Pfeile markieren die Ovarien rechts bzw. links. b Axiale, fettgesättigte T1w Sequenz.

anderen Ursprungs unterscheiden, die an den Uterus angrenzen: Das Leiomyom weist eine vaskuläre Brücke auf, die anderen Tumoren nicht $[30,67]$. Liegt eine Stieldrehung vor, ist zuerst der venöse Abfluss behindert. Die obstruierten Venen können als perifokales Halo mit hypointensem Signal auf T2w und mit hyperintensem Signal auf T1w Aufnahmen abgegrenzt werden $[63,68]$. Leiomyome in hyaliner Degeneration sind häufig hypo- bis isointens auf der T1w Sequenz und hypointens gegenüber Myometrium auf der T2w Sequenz [63]. Die hämorrhagische Infarzierung bei der roten Degeneration ist je nach Zeitpunkt der Hämorrhagie z. B. hyperintens auf T1w und T2w Aufnahmen (subakut) (Abb.9) [63,68]. Wie von intrakraniellen Blutungen bekannt, wechselt auch das MR-Bild extra- 
Abb. 9 Torsion eines subserösen Leiomyoms. Der Pfeil zeigt auf die in der T2w Sequenz isointense bzw. gemischt hyperund hypoechogene Raumforderung kranial des Uterus, dem torquierten Leiomyom entsprechend. Die Pfeilspitze zeigt auf eine Ovula Nabothi. a Sagittale, T2w Sequenz. b Transabdomineller Ultraschall.

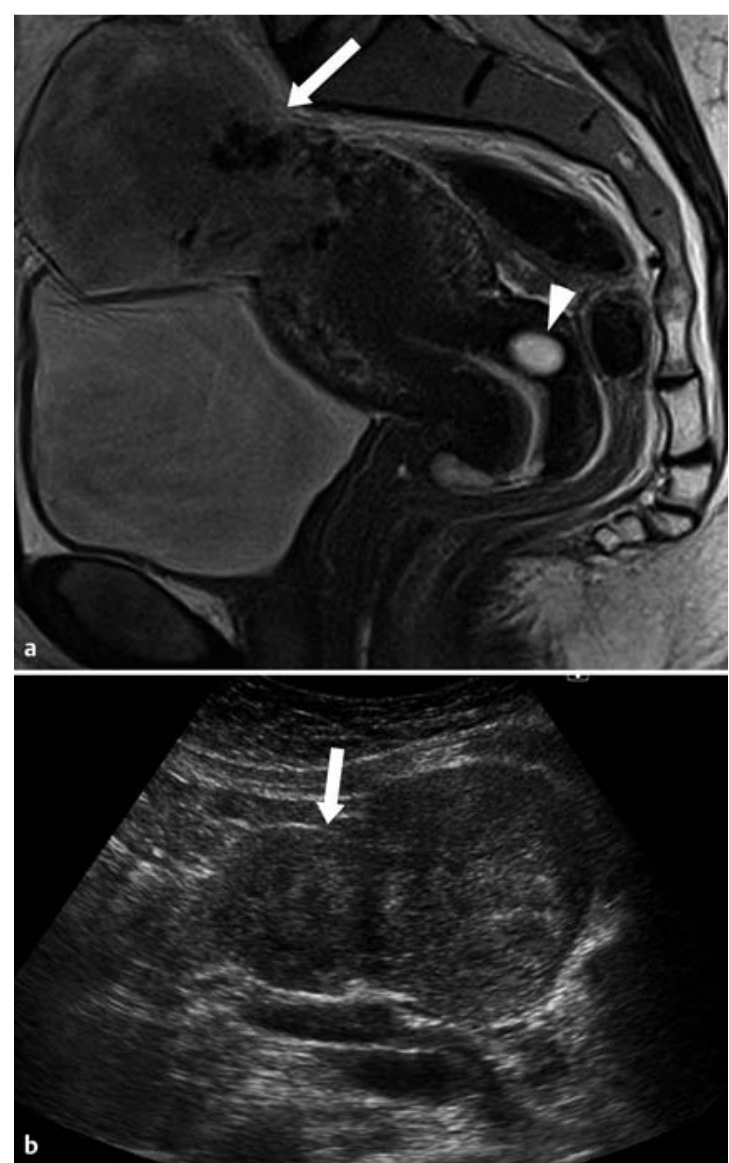

kranieller Blutungen im Lauf der Zeit. Die Morphologie extrakranieller Blutungen folgt dabei aber insgesamt weniger schematischen Abläufen und ist zudem auch heterogener [69].

In der MRT hängt die Darstellung der Infarzierung vom Zeitpunkt der Hämorrhagie ab. Im subakuten Stadium ist sie $z$. B. hyperintens auf T1w und T2w Aufnahmen. Das Signalverhalten der extrakraniellen Hämorrhagien ist jedoch insgesamt heterogener als bei intrakraniellen Blutungen.

\section{Uterusruptur}

Die Uterusruptur ist definiert als Riss von Myometrium und Serosa. Demgegenüber ist bei einer Uterusdehiszenz die Serosa noch intakt. Zumeist basiert eine intrapartale Ruptur auf einer vorbestehenden Narbe des Myometriums, beispielsweise nach einer Sectio caesarea. Eine Ruptur ohne vorherige Narbe ist z. B. Folge eines Traumas oder einer kongenitalen Schwäche des Myometriums (z. B. bei Ehlers-Danlos-Syndrom Typ IV) $[70,71]$. Andere Ursachen sind ein protrahierter
Geburtsvorgang oder stark uterotonische Medikamente [72]. Die Symptome einer Uterusruptur sind unspezifisch: Es kann zu plötzlichem Schmerz zwischen den Wehen, exzessiver vaginaler Blutung mit Schockzeichen, Verlust des Uterustonus und zu Zeichen des fötalen Stresses kommen. Die Uterusdehiszenz verläuft dagegen zumeist asymptomatisch, gelegentliche Symptome sind Dysmenorrhö, Dyspareunie, chronische Unterleibsschmerzen und intermenstruelles „Spotting“ [73].

Man unterscheidet die Uterusruptur von der -dehiszenz: Bei der Dehiszenz ist die Serosa noch intakt, während bei der Ruptur eine Verbindung zwischen Cavitas uteri und Peritonealhöhle besteht. Die Dehiszenz kann konservativ behandelt werden, die Ruptur muss i.d. R. operiert werden.

Ultraschall. Der US ist prognostisch hilfreich, um das Rupturrisiko bei einer zukünftigen Vaginalgeburt nach einer Sectio caesarea zu beurteilen: Liegen der Durchmesser des Uterus unter 3,5 mm oder der des Myometriums unter $2 \mathrm{~mm}$, ist dieses Risiko signifikant erhöht [5]. Zu den US-Befunden einer intrapartalen Uterusruptur gehören das intraamniotische und/oder extrauterine Hämatom und extrauterine fötale Körperteile. Postpartal kann die Disruptionsstelle mittels US nicht identifiziert werden, und Befunde sind eher unspezifisch $[5,73]$.

CT. In der CT gibt es eine Überschneidung mit den zu erwartenden normalen postpartalen Befunden. Bei einer Uterusdehiszenz finden sich nur unspezifische Befunde wie freie Flüssigkeit/Luft, Pleuraerguss oder Darmatonie. Eine Diskontinuität des Myometriums ist ein normaler Befund unmittelbar postpartal. Warnzeichen einer Uterusdehiszenz sind ein „flap“-Hämatom von mehr als $5 \mathrm{~cm}$ und „Straßen“ freier Flüssigkeit/Luft mit Verlauf nach intraperitoneal $[73,74]$. Ein „flap“Hämatom bezeichnet das im Rahmen einer Sectio caesarea entstehende Hämatom an der peritonealen Inzisionsstelle zwischen Blase und Myometrium. Bei einer Ruptur kommuniziert das Cavitas uteri mit der Peritonealhöhle, sodass sich intraperitoneal Luft ansammeln kann. Weitere, eher unspezifische Warnzeichen einer Ruptur sind größere Mengen eines Hämatoperitoneums oder ein infiziertes „flap“-Hämatom. Das „flap“Hämatom ist durch randständige Kontrastmittelaufnahme, freie Luft im Hämatom und interne Septierungen gekennzeichnet [74].

Freie intraabdominelle Luft präpartal weist auf eine Uterusruptur hin. 
MRT. Die MRT ist die Methode mit der höchsten Aussagekraft. Sie kann zwischen antepartaler Uterusruptur, Uterusdehiszenz und uterinen Sakkulationen bei funktioneller Schwäche des Myometriums unterscheiden. Amnionflüssigkeit und periuterine Hämorrhagien stellen sich iso-/hypointens auf T1w und hyperintens auf T2w Aufnahmen dar [5,73]. Die Serosa ist bei der Dehiszenz noch abgrenzbar und hindert ein "flap“Hämatom an der Ausbreitung. Bei der Ruptur erscheint die Serosa dagegen in der fettgesättigten, T2w Sequenz nicht mehr kontinuierlich [74]. Die Unterscheidung von Dehiszenz und Ruptur ist klinisch von großer Relevanz, weil die Dehiszenz konservativ therapiert werden kann, während die Ruptur üblicherweise operiert werden muss. Allerdings korrelieren die Befunde von CT und MRT bei der Differenzierung von Dehiszenz und Ruptur nur schlecht mit dem operativen Befund (Abb. 10) [74].

Die MRT erlaubt in der fettgesättigten T2w Sequenz die Abgrenzung der Serosa und ist deshalb die sensitivere Methode zur Differenzierung zwischen Dehiszenz und Ruptur.

\begin{abstract}
Acute abdominal pain related to the female genital organs is frequently encountered in the emergency department. Gynecological emergencies are diseases of the female reproductive system that are potentially life-threatening and peril the sexual function and fertility. In the diagnostic work-up of acute abdominal pain, a wide variety of differential diagnoses needs to be considered depending on the age of the patient and a concomitant pregnancy. There is significant clinical overlap with gastrointestinal emergencies. Therefore, imaging plays a key role in diagnosing the cause of the pain and the planning of the therapy. The aim of this review is to illustrate the significant role of imaging in frequently encountered gynaecologic emergencies.
\end{abstract}

\section{Keywords}

Emergency · gynaecology · imaging · ovary · uterus

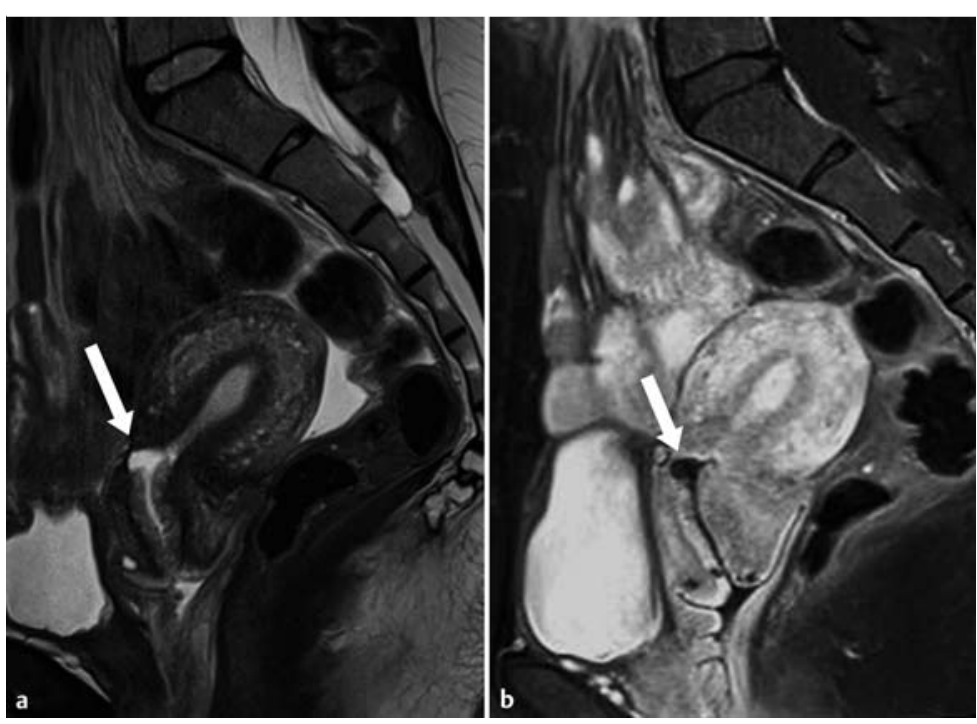

Abb. 10 Uterusdehiszenz. Der Pfeil weist auf das Hämatom bei fokalem Riss des Myometriums und noch intakter Serosa. a Sagittale, T2w Sequenz. b Sagittale, kontrastmittelverstärkte, fettgesättigte T1w Sequenz.

\section{Kernaussagen}

- Wenn die CT bei bei Kindern, Schwangeren oder Frauen im gebärfähigen Alter zum Einsatz kommt, sollte die Strahlendosis besonders beachtet werden. Es gilt das ALARA-Prinzip, d.h. die Dosis sollte „as low as reasonably achievable" sein.

- Die MRT dient zur Abklärung subakuter Krankheitsbilder und unklarer sonografischer Befunde. Bei eindeutiger Indikation und positivem Nutzen-Risiko-Verhältnis kann die MRT auch bei Schwangeren durchgeführt werden.

- Der Hämatometrokolpos (distendierter, blutgefüllter Uterus und Vagina) ist in zwei Drittel aller Fälle durch die Hymenalatresie verursacht. Nach der initialen Abklärung mittels Ultraschall sollte eine assoziierte Nierenfehlbildung und eine Hydronephrose ausgeschlossen werden.
- Die EUG ist der häufigste Notfall in der frühen Schwangerschaft und manifestiert sich i.d. R. in der Tuba uterina. Risikofaktoren sind u. a. „pelvic inflammatory disease“, Endometriose und Voroperationen. Ultraschallzeichen sind ein „blob-sign“ und ein „tubal ring-sign“. Die MRT dient lediglich zur Abklärung unklarer Fälle.

- Leiomyome sind die häufigsten gynäkologischen Neoplasien. Die rote Degeneration des Leiomyoms ist eine wichtige Differenzialdiagnose des akuten Abdomens während der Schwangerschaft. Die Torsion eines Leiomyoms kann ebenfalls eine Ursache für ein akutes Abdomen sein. 


\section{Über die Autoren}

\section{Matthias W. Wagner}

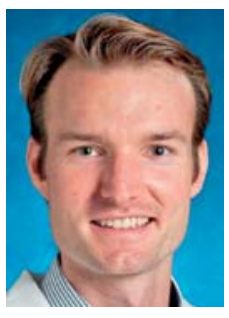

Dr. med. 2006 - 2012 Studium der Medizin an den Universitäten Gießen, München und Kyoto. 2012-2013 Assistenzarzt am Institut für Radiologie, Kantonsspital Baden. 20142015 Research Fellowship - Pediatric Neuroradiology, Johns Hopkins Hospital, Baltimore. Seit Mai 2015 Assistenzarzt am Institut für Diagnostische und Interventionelle Radiologie, UniversitätsSpital Zürich und Adjunct Research Associate am Johns Hopkins Hospital. Forschungsschwerpunkte: Radiogenomics, Texturanalyse und quantitatives Imaging.

\section{Thierry A. G. M. Huisman}

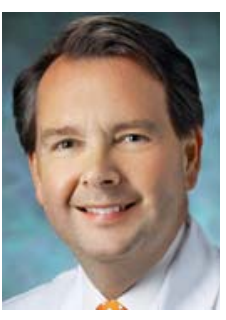

Prof. Dr. med. Chefarzt des Department of Imaging and Imaging Science am Johns Hopkins Bayview Medical Center sowie Chefarzt der Abteilung Pädiatrische Radiologie und Pädiatrische Neuroradiologie am Johns Hopkins Hospital, Baltimore, USA. Thierry Huisman ist Professor für Radiologie, Pädiatrie, Neurologie und Neurochirurgie. Bevor er an das Johns Hopkins Hospital berufen wurde, war er Chefarzt der Abteilung Bilddiagnostik des Universitäts-Kinderspitals Zürich, Schweiz.

\section{Rahel A. Kubik}

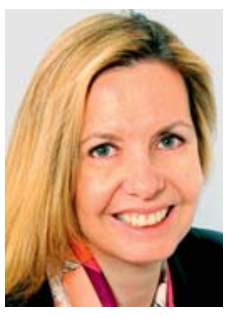

Prof. Dr. med., MPH. 1985-1991 Studium der Humanmedizin und Doktorat an der Universität Zürich, Schweiz. 1992 - 1997 Facharztausbildung Radiologie am Institut für Diagnostische Radiologie, Universitätsspital Zürich sowie am Rockefeller University Hospital, New York, USA. 1999 Habilitation. 2004 Diplom Master of Public Health (interuniversitäres Nachdiplomstudium Universitäten Zürich, Bern, Basel). Seit 2005 Chefärztin des Instituts für Radiologie und Direktorin des Departements Medizinische Dienste, Kantonsspital Baden AG. Mitglied der Geschäftsleitung. 2005 Titularprofessur für Diagnostische Radiologie, Universität Zürich. Klinischer und wissenschaftlicher Schwerpunkt im Bereich gynäkologischer Bildgebung.

\section{Korrespondenzadresse}

Prof. Dr. med. Rahel A. Kubik

$\mathrm{MPH}$, Institut für Radiologie

Kantonsspital Baden AG

Im Ergel 1

$\mathrm{CH}-5404$ Baden

Tel. +41564863802

Fax: +41564863809

E-Mail: rahel.kubik@ksb.ch

\section{Danksagung}

Wir bedanken uns bei Herrn Dr. J. Fröhlich, Medical Director von Guerbet AG und ETH-Dozent, Schweiz, für die kritische Durchsicht der Informationen über Kontrastmittel und bei Herrn Prof. Dr. M. Hohl, Kantonsspital Baden, Schweiz, für die Bereitstellung von Abb. 3 b.

Interessenkonflikt: kein Interessenkonflikt angegeben

\section{Literatur}

1 Roche O, Chavan N, Aquilina J et al. Radiological appearances of gynaecological emergencies. Insights Imaging 2012; 3 : 265-275

2 Tirada N, Dreizin D, Khati NJ et al. Imaging Pregnant and Lactating Patients. Radiographics 2015; 35: 1751- 1765

3 Oto A, Ernst RD, Ghulmiyyah LM et al. MR imaging in the triage of pregnant patients with acute abdominal and pelvic pain. Abdom Imaging 2009; 34: 243-250

4 Atri M, Leduc C, Gillett $P$ et al. Role of endovaginal sonography in the diagnosis and management of ectopic pregnancy. Radiographics 1996; 16: 755-774

5 Masselli G, Derchi L, McHugo J et al. Acute abdominal and pelvic pain in pregnancy: ESUR recommendations. Eur Radiol 2013; 23: $3485-3500$

6 Molins IG, Font JM, Alvaro JC et al. Contrast-enhanced ultrasound in diagnosis and characterization of focal hepatic lesions. World J Radiol 2010; 2: 455-462

7 Nolsoe CP, Lorentzen T. International guidelines for contrastenhanced ultrasonography: ultrasound imaging in the new millennium. Ultrasonography 2016; 35: 89-103

8 Frohlich JM, Kubik-Huch RA. Radiographic, MR or ultrasound contrast media in pregnant or breast-feeding women: what are the key issues? Rofo 2013; 185: 13-25

9 Kubik-Huch RA, Gottstein-Aalame NM, Frenzel T et al. Gadopentetate dimeglumine excretion into human breast milk during lactation. Radiology 2000; 216: 555- 558

10 Posner JC, Spandorfer PR. Early detection of imperforate hymen prevents morbidity from delays in diagnosis. Pediatrics 2005; 115: $1008-1012$ 
11 Basaran M, Usal D, Aydemir C. Hymen sparing surgery for imperforate hymen: case reports and review of literature. J Pediatr Adolesc Gynecol 2009; 22: e61 - 64

12 Fischer JW, Kwan CW. Emergency point-of-care ultrasound diagnosis of hematocolpometra and imperforate hymen in the pediatric emergency department. Pediatr Emerg Care 2014; 30: $128-130$

13 Dane C, Dane B, Erginbas M et al. Imperforate hymen-a rare cause of abdominal pain: two cases and review of the literature. J Pediatr Adolesc Gynecol 2007; 20: 245-247

14 Drakonaki EE, Tritou I, Pitsoulis G et al. Hematocolpometra due to an imperforate hymen presenting with back pain: sonographic diagnosis. J Ultrasound Med 2010; 29: 321 - 322

15 Poll LW, Flake P. Images in clinical medicine. Imperforate hymen with hematocolpometra. N Engl J Med 2011; 365: 157

16 Krafft C, Hartin CWJr, Ozgediz DE. Magnetic resonance as an aid in the diagnosis of a transverse vaginal septum. J Pediatr Surg 2012; 47: $422-425$

17 Beranger-Gibert S, Sakly H, Ballester $\mathrm{M}$ et al. Diagnostic Value of MR Imaging in the Diagnosis of Adnexal Torsion. Radiology 2016; 279: $461-470$

18 Damigos E, Johns J, Ross J. An update on the diagnosis and management of ovarian torsion. The Obstetrician \& Gynaecologist 2012; 14: $229-236$

19 Hibbard LT. Adnexal torsion. Am J Obstet Gynecol 1985; 152: $456-461$

20 Duigenan S, Oliva E, Lee SI. Ovarian torsion: diagnostic features on CT and MRI with pathologic correlation. AJR Am J Roentgenol 2012; 198: W122-131

21 Chang HC, Bhatt S, Dogra VS. Pearls and pitfalls in diagnosis of ovarian torsion. Radiographics 2008; 28: 1355-1368

22 Pena JE, Ufberg D, Cooney $\mathrm{N}$ et al. Usefulness of Doppler sonography in the diagnosis of ovarian torsion. Fertil Steril 2000; 73: 1047 - 1050

23 Chiou SY, Lev-Toaff AS, Masuda E et al. Adnexal torsion: new clinical and imaging observations by sonography, computed tomography, and magnetic resonance imaging. J Ultrasound Med 2007; 26: 1289-1301

24 Wilkinson C, Sanderson A. Adnexal torsion - a multimodality imaging review. Clin Radiol 2012; 67: 476-483

25 Lee EJ, Kwon HC, Joo HJ et al. Diagnosis of ovarian torsion with color Doppler sonography: depiction of twisted vascular pedicle. J Ultrasound Med 1998; 17: $83-89$

26 Albayram F, Hamper UM. Ovarian and adnexal torsion: spectrum of sonographic findings with pathologic correlation. J Ultrasound Med 2001; 20: 1083 - 1089

27 Lourenco AP, Swenson D, Tubbs RJ et al. Ovarian and tubal torsion: imaging findings on US, CT, and MRI. Emerg Radiol 2014; $21: 179-187$

28 Rha SE, Byun JY, Jung SE et al. CT and MR imaging features of adnexal torsion. Radiographics 2002; 22: 283 - 294

29 Hiller N, Appelbaum L, Simanovsky N et al. CT features of adnexal torsion. AJR Am J Roentgenol 2007; 189: 124 - 129

30 Rajkotia K, Veeramani M, Macura KJ. Magnetic resonance imaging of adnexal masses. Top Magn Reson Imaging 2006; 17: $379-397$

31 Moribata Y, Kido A, Yamaoka T et al. MR imaging findings of ovarian torsion correlate with pathological hemorrhagic infarction. Journal of Obstetrics and Gynaecology Research 2015; $41: 1433-1439$
32 Hiller N, Sella T, Lev-Sagi A et al. Computed tomographic features of tuboovarian abscess. J Reprod Med 2005; 50: 203 208

33 Jeong WK, Kim Y, Song SY. Tubo-ovarian abscess: CT and pathological correlation. Clin Imaging 2007; 31: 414-418

34 Lee DC, Swaminathan AK. Sensitivity of ultrasound for the diagnosis of tubo-ovarian abscess: a case report and literature review. J Emerg Med 2011; 40: 170 - 175

35 Wilbur AC, Aizenstein RI, Napp TE. CT findings in tuboovarian abscess. AJR Am J Roentgenol 1992; 158: 575-579

36 Ellis JH, Francis IR, Rhodes $M$ et al. CT findings in tuboovarian abscess. J Comput Assist Tomogr 1991; 15: 589 - 592

37 Eshed I, Halshtok O, Erlich Z et al. Differentiation between right tubo-ovarian abscess and appendicitis using CT - a diagnostic challenge. Clin Radiol 2011; 66: 1030-1035

38 Noone TC, Semelka RC, Worawattanakul S et al. Intraperitoneal abscesses: diagnostic accuracy of and appearances at MR imaging. Radiology 1998; 208: 525 - 528

39 Dohke M, Watanabe Y, Okumura A et al. Comprehensive MR imaging of acute gynecologic diseases. Radiographics 2000; 20: $1551-1566$

40 Kim SH, Kim SH, Yang DM et al. Unusual causes of tubo-ovarian abscess: CT and MR imaging findings. Radiographics 2004: 24: 1575 - 1589

41 Bakir B, Bakan S, Tunaci M et al. Diffusion-weighted imaging of solid or predominantly solid gynaecological adnexial masses: is it useful in the differential diagnosis? Br J Radiol 2011; 84: $600-611$

42 Fujii S, Kakite S, Nishihara K et al. Diagnostic accuracy of diffusion-weighted imaging in differentiating benign from malignant ovarian lesions. J Magn Reson Imaging 2008; 28: 1149 1156

43 Rezvani M, Shaaban AM, Kennedy AM. The role of multimodality imaging after cesarean delivery. Ultrasound Q 2015; 31 : $5-18$

44 Brown CE, Stettler RW, Twickler D et al. Puerperal septic pelvic thrombophlebitis: incidence and response to heparin therapy. Am J Obstet Gynecol 1999; 181: 143 - 148

45 Josey WE, Staggers SRJr. Heparin therapy in septic pelvic thrombophlebitis: a study of 46 cases. Am J Obstet Gynecol 1974; 120: $228-233$

46 Garcia J, Aboujaoude R, Apuzzio J et al. Septic pelvic thrombophlebitis: diagnosis and management. Infect Dis Obstet Gynecol 2006; 2006: 1 -4

47 Kominiarek MA, Hibbard JU. Postpartum ovarian vein thrombosis: an update. Obstet Gynecol Surv 2006; 61: 337 - 342

48 De Cuyper K, Eyselbergs M, Bernard P et al. Added value of diffusion weighted MR imaging in the diagnosis of postpartum ovarian vein thrombosis. Jbr-btr 2014; 97: 242 - 244

49 Kataoka ML, Togashi K, Kobayashi H et al. Evaluation of ectopic pregnancy by magnetic resonance imaging. Hum Reprod 1999; 14: $2644-2650$

50 Della-Giustina D, Denny M. Ectopic pregnancy. Emerg Med Clin North Am 2003; 21: 565 - 584

51 Tamai K, Koyama T, Togashi K. MR features of ectopic pregnancy. Eur Radiol 2007; 17: 3236 - 3246

52 Refaat B, Dalton E, Ledger WL. Ectopic pregnancy secondary to in vitro fertilisation-embryo transfer: pathogenic mechanisms and management strategies. Reprod Biol Endocrinol 2015; 13: 30 
53 Baron KT, Babagbemi KT, Arleo EK et al. Emergent complications of assisted reproduction: expecting the unexpected.

Radiographics 2013; 33: 229-244

54 Braffman BH, Coleman BG, Ramchandani P et al. Emergency department screening for ectopic pregnancy: a prospective US study. Radiology 1994; 190: 797-802

55 Condous G, Okaro E, Khalid A et al. The accuracy of transvaginal ultrasonography for the diagnosis of ectopic pregnancy prior to surgery. Hum Reprod 2005; 20: $1404-1409$

56 Shalev E, Yarom I, Bustan M et al. Transvaginal sonography as the ultimate diagnostic tool for the management of ectopic pregnancy: experience with 840 cases. Fertil Steril 1998; 69: $62-65$

57 Brown DL, Doubilet PM. Transvaginal sonography for diagnosing ectopic pregnancy: positivity criteria and performance characteristics. J Ultrasound Med 1994; 13: 259-266

58 Sadek AL, Schiotz HA. Transvaginal sonography in the management of ectopic pregnancy. Acta Obstet Gynecol Scand 1995; 74: 293-296

59 Yoshigi J, Yashiro N, Kinoshita T et al. Diagnosis of ectopic pregnancy with MRI: efficacy of T2*-weighted imaging. Magn Reson Med Sci 2006; 5: 25-32

60 Murase E, Siegelman ES, Outwater EK et al. Uterine leiomyomas: histopathologic features, MR imaging findings, differential diagnosis, and treatment. Radiographics 1999; 19: $1179-$ 1197

61 McLucas B. Diagnosis, imaging and anatomical classification of uterine fibroids. Best Pract Res Clin Obstet Gynaecol 2008; 22: $627-642$

62 Furey EA, Bailey AA, Pedrosa I. Magnetic resonance imaging of acute abdominal and pelvic pain in pregnancy. Top Magn Reson Imaging 2014; 23: 225 - 242

63 Ueda H, Togashi K, Konishi I et al. Unusual appearances of uterine leiomyomas: MR imaging findings and their histopathologic backgrounds. Radiographics 1999; 19: 131-145

64 Potter AW, Chandrasekhar CA. US and CT evaluation of acute pelvic pain of gynecologic origin in nonpregnant premenopausal patients. Radiographics 2008; 28: $1645-1659$

65 Roy C, Bierry G, El Ghali S et al. Acute torsion of uterine leiomyoma: CT features. Abdom Imaging 2005; 30: 120-123
66 Katz DS, Khalid M, Coronel EE et al. Computed tomography imaging of the acute pelvis in females. Can Assoc Radiol J 2013; 64: $108-118$

$67 \mathrm{Kim} \mathrm{JC}$, Kim SS, Park JY. "Bridging vascular sign" in the MR diagnosis of exophytic uterine leiomyoma. J Comput Assist Tomogr 2000; 24: 57-60

68 Nishino M, Hayakawa K, Iwasaku K et al. Magnetic resonance imaging findings in gynecologic emergencies. J Comput Assist Tomogr 2003; 27: $564-570$

69 Grand DJ, Mayo-Smith WW, Woodfield CA. Practical Body MRI: Protocols, Applications and Image Interpretation. Cambridge: Cambridge University Press; 2012

70 Walsh CA, Reardon W, Foley ME. Unexplained prelabor uterine rupture in a term primigravida. Obstet Gynecol 2007; 109: 455

71 Pepin M, Schwarze U, Superti-Furga A et al. Clinical and genetic features of Ehlers-Danlos syndrome type IV, the vascular type. N Engl J Med 2000; 342: 673-680

72 Khabbaz AY, Usta IM, El-Hajj MI et al. Rupture of an unscarred uterus with misoprostol induction: case report and review of the literature. J Matern Fetal Med 2001; 10: 141 - 145

73 Moshiri M, Osman S, Bhargava P et al. Imaging evaluation of maternal complications associated with repeat cesarean deliveries. Radiol Clin North Am 2014; 52: 1117-1135

74 Rodgers SK, Kirby CL, Smith RJ et al. Imaging after cesarean delivery: acute and chronic complications. Radiographics 2012; 32: $1693-1712$

75 Emans SJ et al. Pediatric and Adolescent Gynecology. Baltimore, Md: Lippincott Williams \& Wilkins; 2005: 369

76 Hueppchen N, Ling FW. Ectopic pregnancy and abortion. In: Beckmann CR, Ling FW, Smith RP et al., (eds). Obstetrics and gynecology. 6th ed. Baltimore, Md: Lippincott Williams \& Wilkins; 2010: $142-143$

77 European Society of urogenital radiology. 9.0 Contrast Media Guidelines. Letztes Update: 20.4. 2016. Im Internet: http:// www.esur.org/esur-guidelines; Stand: 30.7.2016 


\section{CME-Fragen}

Die folgenden Fragen beziehen sich auf den vorangehenden Beitrag. Bitte schicken Sie uns die entsprechenden Lösungsbuchstaben. Jeweils eine Antwort ist richtig. Die Vergabe von CME-Punkten ist an die korrekte Beantwortung der Multiple-Choice-Fragen gebunden.

1

Welche der folgenden Aussagen zu gestielten subserösen Leiomyomen ist nicht richtig?
A Submuköse und subseröse Leiomyome neigen zu gestieltem Wachstum mit erhöhtem Risiko für eine Torsion.

B Die MRT ist hilfreich, um die uterine Anheftungsstelle zu finden.

C Nekrotische und hämorrhagische infarzierte Leiomyome erscheinen in der nativen CT immer hypodens.

D Der Pedikel ist häufig dünn und mit dem US nicht einsehbar.

E Leiomyome sind die häufigste gynäkologische Neoplasie.
Welche der folgenden Aussagen zum Hämatometrokolpos ist nicht richtig?
A Etwa zwei Drittel sind Folge einer Hymenalatresie.

B Die Diagnose wird zumeist im Rahmen der Pränataldiagnostik, direkt nach der Geburt oder mit Beginn der Pubertät gestellt.

C Computertomografisch ist der HMK eine solide, kontrastmittelaufnehmende Raumforderung.

D Die MRT wird zur Abklärung komplexer Fehlbildungen benötigt.

E Ursachen für den HMK sind u.a. kloakale Fehlbildungen und Anomalien der Müller-Gänge.
Welche der folgenden Aussagen zu Leiomyomen ist richtig?
A Die rote Degeneration ist die häufigste Degenerationsvariante.

B Die hyaline Degeneration ist hyperintens auf T1w und T2w Aufnahmen.

C Bei einer Stieldrehung kommt es zuerst zur Obstruktion der arteriellen Versorgung.

D Die rote Degeneration tritt häufiger in der Schwangerschaft auf.

E Eine hämorrhagische Infarzierung des stielgedrehten Leiomyoms erscheint in der nativen CT hypodens.

4

Welche der folgenden Aussagen zu ionisierenden Strahlen in der Schwangerschaft ist richtig?
A Bei Notfällen muss der Strahlenbelastung durch die CT keine Beachtung geschenkt werden.

B Eine Ein-Phasen-CT erreicht üblicherweise teratogene Strahlenwerte.

C Eine strenge Nutzen-Risiko-Analyse für die Mutter und den Fötus sollte einer Entscheidung zur CT-Untersuchung vorausgehen.

D Das ALARA-Prinzip gilt nur für die MRT.

E Die CT sollte als Suchmethode bei unklaren Befunden eingesetzt werden.

\section{5}

Welche der folgenden Aussagen zum Hämatometrokolpos ist nicht richtig?
A Der Hämatometrokolpos tritt bei Teenagern am häufigsten auf.

B Typische Symptome sind Bauchmerzen in Verbindung mit primärer Amenorrhö.

C Bei ausgeprägten Befunden kann auch eine Hydro-/Hämatosalpinx auftreten.

D Die Abklärung erfolgt initial mit der CT.

E Es sollte immer auch nach einer assoziierten Nierenfehlbildung oder einer Hydronephrose gesucht werden.

\section{6}

Welche der folgenden Aussagen zu Adnextorsion ist nicht richtig?
A Brenner-Tumoren sind eine häufige Ursache der Torsion.

B Die Torsion der Adnexe ist ein seltener Notfall.

C Für den Erhalt der Fertilität ist eine frühe Diagnose entscheidend.

D Mehrheitlich tritt die Torsion rechts auf und betrifft sowohl Ovarien als auch Tuba uterina.

E Ein „Whirlpool“-Zeichen kann sowohl im US als auch in der MRT auftreten. 


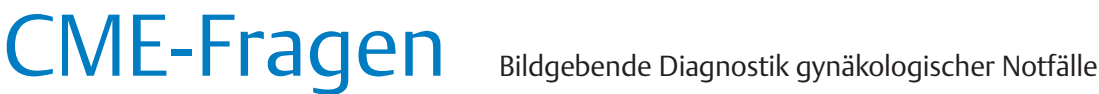

7

Welche der folgenden Aussagen zur EUG ist nicht richtig?
A Sie ist der häufigste Notfall in der frühen Schwangerschaft.
B Nahezu alle EUGs treten in der Cervix uteri auf.
C „Blob“-Zeichen und „tubal-ring“-Zeichen im US sind typische Zeichen einer EUG.
D Die assoziierte Hämatometra stellt sich iso-/hyperintens auf T1w und mit gemischtem Signal auf T2w Aufnahmen dar.
E Zu den Risikofaktoren gehören PID, Endometriose, Voroperationen und späte Primiparität.

\section{8}

Welche der folgenden Aussagen zur Uterusruptur ist richtig?
A Sie ist eine häufige Komplikation während der Schwangerschaft.
B Der US findet keine Anwendung bei der Uterusruptur, weil das Hämatom das Bild trübt.
C Die MRT erlaubt die Differenzierung zwischen Uterusdehiszenz und -ruptur.
D Die Diskontinuität des Myometriums weist unmittelbar postpartal auf eine Ruptur hin.
E Eine Ruptur entsteht i.d.R. „de-novo“.

Welche der folgenden Aussagen zu „pelvic inflammatory disease“ und Tuboovarialabszess ist nicht richtig?
A Der TOA kann eine Folge einer ungenügenden Behandlung einer PID sein.
B Die PID wird durch eine aszendierende Infektion von Chlamydien oder Neisserien verursacht.
C Der TOA erscheint in der CT als wandverdickte Kollektionen mit randständiger Kontrastmittelaufnahme.
D Der TOA lässt sich in der MRT am besten auf der kontrastmittelverstärkten, fettgesättigten, T1w Sequenz erkennen.
E Der US hat für den TOA eine geringe Sensitivität und Spezifität.

\section{0}

Welche der folgenden Aussagen zur Bildgebung während der Schwangerschaft ist richtig?
A Die Durchführung einer MRT setzt die vorherige Abklärung mittels CT voraus.

B Gadoliniumhaltiges Kontrastmittel kann unter eingeschränkten Bedingungen (bei eindeutig positivem Nutzen-Risiko-Verhältnis und vorheriger Patientenaufklärung) eingesetzt werden.

C Nach Applikation eines gadoliniumhaltigen Kontrastmittels sollte nach der Geburt auf eine neonatale Hypothyreose geprüft werden.

D Die MRT ist die Methode der Wahl zur initialen Abklärung schwerer Traumata.

E US-Kontrastmittel werden häufig bei Schwangerschaftskomplikationen eingesetzt.

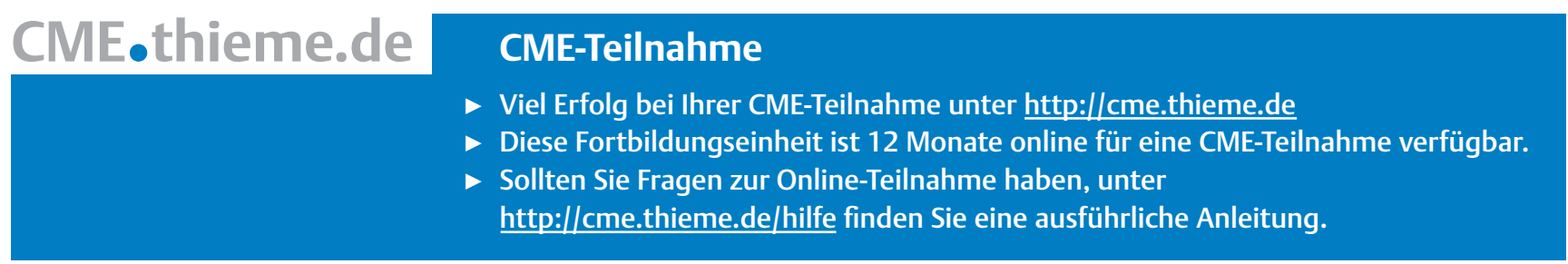

